\title{
Effect of Comprehensive Nursing Intervention on Thyroid Stimulating Hormone Suppression for Patients with Differentiated Thyroid Cancer
}

\author{
Zhou Miaoli, Lin Qingran, Shang Jingjie, Gong Jian, Xu Hao \\ Department of Nuclear Medicine, The First Affiliated Hospital of Jinan University, Guangzhou, China
}

Email address:

zhoumiaoli2018@sina.com (Zhou Miaoli)

\section{To cite this article:}

Zhou Miaoli, Lin Qingran, Shang Jingjie, Gong Jian, Xu Hao. Effect of Comprehensive Nursing Intervention on Thyroid Stimulating Hormone Suppression for Patients with Differentiated Thyroid Cancer. American Journal of Nursing Science. Vol. 8, No. 1, 2019, pp. 32-35. doi: 10.11648/j.ajns.20190801.15

Received: December 2, 2018; Accepted: January 31, 2019; Published: February 27, 2019

\begin{abstract}
Objective to investigate the effect of comprehensive nursing intervention on thyroid stimulating hormone (TSH) suppression for patients with differentiated thyroid cancer (DTC) in the Department of Nuclear Medicine. Methods From January 2016 to December 2016, a total of 90 patients with DTC, receiving ${ }^{131}$ I treatment in the Department of Nuclear Medicine of the First Affiliated Hospital of Jinan University are enrolled in this study. All patients have received TSH suppression treatment for at least 3 months prior to admission, and are given individual-based comprehensive nursing intervention on TSH suppression after admission. Through questionnaire and telephone follow-up, patients' awareness of TSH suppression and their medication compliance before and after nursing intervention are analyzed and compared. Results The awareness of the knowledge of TSH suppression and medication compliance are significantly improved in 90 patients after comprehensive nursing intervention $(\mathrm{P}<0.05)$. Conclusions Individual-based comprehensive nursing intervention can effectively improve awareness of the knowledge of TSH suppression, medication compliance and confidence in cure in patients with DTC, which helps promote the harmonious relationship between nurses and patients and improve the prognosis of patients. The comprehensive nursing intervention is worthy of clinical application.
\end{abstract}

Keywords: Differentiated Thyroid Cancer, TSH Suppression, Nursing Intervention

\section{Introduction}

At present, a combination of operation, ${ }^{131}$ I treatment and TSH suppression is the recognized treatment for differentiated thyroid cancer [1]. TSH suppression refers to the condition of giving exogenous thyroid hormone to patients with thyroid cancer after operation and ${ }^{131} \mathrm{I}$ treatment to suppress their thyroid stimulating hormone (TSH) below the normal TSH range so as to supplement thyroid hormone and to inhibit the growth of DTC cells. TSH suppression can significantly reduce the risk of metastasis and recurrence of thyroid cancer to improve patients' survival rate and quality of life [2]. But clinically, some patients pay insufficient attention to TSH suppression and lack scientific knowledge of taking exogenous thyroid hormone. Therefore, TSH suppression and individual-based comprehensive nursing intervention have been given to the post-DTC operation patients in the
Department of Nuclear Medicine from January to December 2016, and satisfying results have been achieved, which are reported as follows.

\section{Participants and Methods}

\subsection{Participants}

Ninety post-DTC operation patients who were receiving ${ }^{131} \mathrm{I}$ treatment in the Department of Nuclear Medicine of the First Affiliated Hospital of Jinan University from January to December 2016 were included. The patients, consisting of 25 males $(27.8 \%)$ and 65 females $(72.2 \%)$, aged from 19 to 66 , with an average age of $39.23 \pm 14.57$. There were 87 cases of papillary thyroid carcinoma and 3 cases of follicular thyroid 
carcinoma. All the patients have received TSH suppression for at least 3 months after operation and have stopped taking exogenous thyroid hormone for 3 to 4 weeks to receive ${ }^{131} \mathrm{I}$ treatment before admission to the hospital.

\subsection{Methods}

\subsubsection{Personalized Instruction of TSH Suppression-Related Knowledge During Hospitalization}

Leaflets on TSH suppression and one-to-one personalized instruction were given by in-charge nurses to all the participants. The instruction included: a. the importance of TSH suppression; $b$. the best ways to take thyroid hormone and matters needing attention; c. hypothyroidism and its hazards; $d$. the appropriate time to resume TSH suppression and the initial dose of thyroid hormone; e. side effects of long-term TSH suppression; f. the importance of medication compliance and regular reexamination during thyroid hormone treatment.

\subsubsection{Mental Nursing Intervention}

Nursing staff imparted the knowledge of disease and treatment in an easy-to-understand way, with careful and patient explanation, paid attention to the patient's feedback and questions, and gave timely answers. More comfort and encouragement were given, and successful cases and rehabilitative cases were introduced to enhance patients' confidence in treatment. Patients were informed of the hotline and WeChat platform of the department for their convenience of consulting the nursing staff to relieve psychological barriers. In addition, when intensive instruction cause in some patients psychological burden that the previous improper medication method may affect the prognosis, psychological intervention should be taken to relieve their burden. If necessary, the patients can be referred to the psychologist for further consultation and treatment.

\subsubsection{Continuous Post-Discharge Nursing Intervention}

A patient information database was established, and individual-based integrated management and continuous nursing intervention were carried out according to the patient's condition and clinical manifestation. The continuous nursing intervention included: a. instruction on the details of the subsequent visit and SMS reminder three days prior to the subsequent visit; b. monthly telephone follow-up on medication compliance, uncomfortable situation, sleep and diet, and guidance on how to take medicine correctly and regularly; c. guidance for family members of patients at younger age, at lower education level or having language communication barrier to strengthen supervision so as not to delay or affect the treatment; d. making a good record of each follow-up for better continuous follow-ups.

\subsection{Evaluation}

Self-designed questionnaires were used to investigate patients' awareness of the knowledge of TSH suppression and their medication compliance before and after comprehensive nursing intervention. The questionnaire survey was conducted on the day of admission, the day of discharge, and on the third month after discharge.

\subsection{Statistical Methods}

The data was processed in the statistical software SPSS 17.0. The measurement data was presented as mean \pm standard deviation $(\bar{x} \pm s)$ and analyzed by chi-square test. When $\alpha=0.05$ and $\mathrm{P}<0.05$, there was significant difference.

\section{Results}

\subsection{The Comparison of Patients' Awareness of TSH Suppression-Related Knowledge Before and After Comprehensive Nursing Intervention}

Patients' awareness of the importance of TSH suppression, knowledge of the best ways to take thyroid hormone drugs and matters needing attention, hypothyroidism and its hazards, and side effects of TSH suppression and regular reexamination were significantly improved. And there was significant difference before and after comprehensive nursing intervention.

Table 1. Comparison of the awareness of TSH suppression-related knowledge before and after intervention ( $n=90)$

\begin{tabular}{|c|c|c|c|c|}
\hline Measurements & $\begin{array}{l}\text { Number of patients before } \\
\text { intervention }\end{array}$ & $\begin{array}{l}\text { Number of patients after } \\
\text { intervention }\end{array}$ & $\chi^{2}$ & $\boldsymbol{P}$ \\
\hline Importance of TSH suppression & $63(70.0 \%)$ & $87(96.7 \%)$ & 22.042 & 0.000 \\
\hline Knowledge of the best ways to take thyroid hormone drugs & $77(85.6 \%)$ & $89(98.9 \%)$ & 10.083 & 0.000 \\
\hline Knowledge of hypothyroidism and its hazards & $43(47.8 \%)$ & $84(93.3 \%)$ & 41.000 & 0.000 \\
\hline Side effects of TSH suppression & $25(27.8 \%)$ & $83(92.2 \%)$ & 58.000 & 0.000 \\
\hline Importance of regular reexamination & $21(23.3 \%)$ & $88(97.8 \%)$ & 67.000 & 0.000 \\
\hline
\end{tabular}

$* P<0.05$ indicates that there is significant difference in patients' awareness of TSH suppression-related knowledge before and after intervention.

\subsection{Comparison of Patients'Medication Compliance Before and After Comprehensive Nursing Intervention}

Patients achieved remarkable improvement concerning medication compliance and significant difference in medication compliance was found before and after intervention. 
Table 2. Comparison of medication compliance before and after intervention ( $n=90)$.

\begin{tabular}{|c|c|c|c|c|}
\hline Measurements & $\begin{array}{l}\text { Number of patients before } \\
\text { intervention }\end{array}$ & $\begin{array}{l}\text { Number of patients after } \\
\text { intervention }\end{array}$ & $\chi^{2}$ & $\boldsymbol{P}$ \\
\hline Time of taking medicine and regular behavior & $72(80.0 \%)$ & $89(98.9 \%)$ & 15.059 & 0.000 \\
\hline Knowledge of correct dose of medication & $81(90.0 \%)$ & $89(98.9 \%)$ & 6.125 & 0.008 \\
\hline Behavior of forgetting taking medicine & $19(21.1 \%)$ & $2(2.2 \%)$ & 15.059 & 0.000 \\
\hline Positive awareness of taking medicine & $75(83.3 \%)$ & $87(96.7 \%)$ & 10.083 & 0.000 \\
\hline
\end{tabular}

${ }^{*} P<0.05$ indicates that there is significant difference in patients' medication compliance before and after intervention.

\section{Discussion}

\subsection{Scientific and Regular Medication and Reexamination to Contribute to TSH Suppression Treatment}

TSH level is an independent predictor of recurrence and death rate of thyroid cancer, and there is a positive correlation between TSH level and thyroid cancer recurrence and mortality [3]. So a reasonable TSH suppression regimen should be developed according to patients' thyroid hormone levels [4-5]. However, the dose of thyroid hormone varies from person to person, so individualized TSH treatment needs to be performed on patients with differentiated thyroid cancer according to TNM staging and risk stratification. Therefore, scientific and regular medication and reexamination become important factors contributing to the TSH suppression treatment. In this study post-DTC operation patients were given comprehensive, standardized and professional nursing care and guidance to not only enhance their knowledge of disease and treatment, but also improve their treatment and medication compliance, which enhance patients' awareness of health management, playing an important role in improving prognosis and health.

\subsection{Individual Health Education to Improve Patients' Knowledge of TSH Suppression Treatment}

TSH suppression indispensable life-long treatment for patients with DTC. Studies have shown that a reasonable and effective one-to-one individual health education can significantly improve patient's knowledge of disease and their treatment compliance [6]. In this study we found most patients pay insufficient attention to TSH suppression treatment and lack knowledge of how to take exogenous thyroid hormone properly and effectively. Besides, patients with DTC may need to take diagnostic 131I whole body scan or receive 131I treatment again during the long-term reexamination, which may involves drug withdrawal and resuming taking medicine. And during the withdrawal, the patients due to hypothyroidism may suffer some discomforts such as decreased work quality, low mood, sub-health, etc., and some may have severe anxiety or even depression [7]. However, supplementation with excessive exogenous thyroid hormone may lead to subclinical hyperthyroidism, symptoms including tremors, anxiety, insomnia, intolerance of heat, palpitations, etc. For elderly patients with cardiovascular disease, it may increase the risk of cardiovascular disease, or may even lead to atrial fibrillation, affecting calcium metabolism in the body. For postmenopausal women it may increase the incidence of osteoporosis [8]. And for women of childbearing age, adequate maternal thyroid hormone during pregnancy is important to ensure the health of the mother and fetus [9-11]. Therefore, it is essential to guide patients to take medication and reexamination correctly and regularly, and to conduct follow-up intervention after discharge and give guidance on continuous treatment. This study focused on the problems patients have and carried out individualized health education, which significantly improved patient's knowledge of TSH suppression treatment, including the significance of TSH inhibition treatment, the best methods of taking thyroid hormone medication, hypothyroidism and its hazards, the side effects of TSH suppression treatment and the significance of regular reexamination, etc., thus enhancing the patient's awareness of the disease and improving their treatment and medication compliance. It also confirmed that cancer patients generally had a strong willingness to improve their health, but they had limited health knowledge and abilities for health practices [12]. So the comprehensive nursing intervention in the radionuclide ward can improve DTC patients' awareness of health management, and encourage them to pay attention to regular reexamination, playing a positive role in improving the prognosis.

\subsection{Continuous Nursing Intervention After Discharge to Ensure Patients to Take Medication in a Correct and Regular Manner}

In TSH inhibition treatment, missing a single dose of medication within a week can result in a $14 \%$ reduction in total dose over the entire week [13]. In the study, we find that $20 \%, 10 \%, 21.1 \%$, and $16.7 \%$ of patients conducted irregular medications, took unclear doses, forgot taking medication and were reluctant to take medication respectively. It is very essential to take thyroid hormone medication regularly on a daily basis to ensure effective plasma concentration. Therefore, we continue to emphasize the importance of taking thyroid hormones regularly in the continuous nursing after discharge, and teach patients to ensure on-time medication by using alarm clocks or mobile phone reminders, taking seven-day fixed kits or asking family members to help remind them, which improve the patient's medication compliance, and reflect the necessary role of continuous nursing intervention for patients with thyroid cancer after surgery.

\section{Conclusions}

In conclusion, the comprehensive nursing intervention for post-DTC operation patients in the radionuclide ward can 
enhance patients' knowledge of disease and treatment, improve their compliance with treatment and medication, and enhance patients' awareness of health management, which helps promote the harmonious relationship between nurses and patients and improve the prognosis of patients. The comprehensive nursing intervention is worthy of clinical application.

\section{References}

[1] Marcelino, Lopes, Madureira, Ferreira, \& Limbert. (2015). Ability of the rhtsh stimulation test to predict relapse in patients with differentiated thyroid carcinoma, after long-term follow-up. Oncology Letters, 9 (3), 1281-1286.

[2] Alexander, E. K., Pearce, E. N., Brent, G. A., Brown, R. S., Chen, H., \& Dosiou, C., et al. (2017). 2017 guidelines of the american thyroid association for the diagnosis and management of thyroid disease during pregnancy and the postpartum. Thyroid, 27 (3), 315-389.

[3] Mcleod, D. S. A., Cooper, D. S., Ladenson, P. W., Ain, K. B., Brierley, J. D., \& Fein, H. G., et al. (2014). Prognosis of differentiated thyroid cancer in relation to serum thyrotropin and thyroglobulin antibody status at time of diagnosis. Thyroid Official Journal of the American Thyroid Association, 24 (1), $35-42$.

[4] Guan, H., Shan, Z., Teng, X., Li, Y., Teng, D., \& Jin, Y., et al. (2010). Influence of iodine on the reference interval of tsh and the optimal interval of tsh: results of a follow-up study in areas with different iodine intakes. Clinical Endocrinology, 69 (1), 136-141.

[5] Ovadia, Y. S., Gefel, D., Turkot, S., Aharoni, D., Fytlovich, S., \& Troen, A. M. (2014). Elevated serum thyroglobulin and low iodine intake are associated with nontoxic nodular goiter among adults living near the eastern mediterranean coast. J Thyroid Res, 2014, 913672.

[6] Moon, J. H., Kim, K. M., Oh, T. J., Choi, S. H., Lim, S., \& Park,
Y. J., et al. (2017). The effect of tsh-suppression on vertebral trabecular bone scores in patients with differentiated thyroid carcinoma. Journal of Clinical Endocrinology \& Metabolism, 102 (1), jc.2016-2740.

[7] Bilezikian, J. P., Brandi, M. L., Cusano, N. E., Mannstadt, M., Rejnmark, L., \& Rizzoli, R., et al. (2016). Management of hypoparathyroidism: present and future. Journal of Clinical Endocrinology \& Metabolism, 101 (6), jc20153910.

[8] Juweid, M. E., Hajjar, G., Swayne, L. C., Sharkey, R. M., Suleiman, S., \& Herskovic, T., et al. (2015). Phase i/ii trial of131i-mn-14 f (ab) 2 anti-carcinoembryonic antigen monoclonal antibody in the treatment of patients with metastatic medullary thyroid carcinoma. Cancer, 85 (8), 1828-1842.

[9] Giacobbe, A. M., Grasso, R., Triolo, O., Tonni, G., \& Granese, R. (2015). Thyroid diseases in pregnancy: a current and controversial topic on diagnosis and treatment over the past 20 years. Archives of Gynecology \& Obstetrics, 292 (5), 995-1002.

[10] Dosiou, C., Barnes, J., Schwartz, A., Negro, R., Crapo, L., \& Stagnarogreen, A. (2012). Cost-effectiveness of universal and risk-based screening for autoimmune thyroid disease in pregnant women. J Clin Endocrinol Metab, 97 (97), 1536-1546.

[11] Mannisto T, Vanmma ki M, Pouta A, et al. Thyrdd dysfunction and autoantibodies during pregnancy as predictive factors of pregnancy complication and maternal morbilith in later life. $\mathrm{J}$ Clin Endocfinol Metab, 2010, 95 (3): 1084-1094.

[12] Hoftijzer, H. C., Heemstra, K. A., Corssmit, E. P., Aa, V. D. K., Romijn, J. A., \& Smit, J. W. (2008). Quality of life in cured patients with differentiated thyroid carcinoma. Journal of Clinical Endocrinology \& Metabolism, 93 (1), 200-3.

[13] Juweid, M. E., Hajjar, G., Swayne, L. C., Sharkey, R. M., Suleiman, S., \& Herskovic, T., et al. (2015). Phase i/ii trial of131i-mn-14 $\mathrm{f}$ (ab) 2 anti-carcinoembryonic antigen monoclonal antibody in the treatment of patients with metastatic medullary thyroid carcinoma. Cancer, 85 (8), 1828-1842. 